\title{
Pathogen-Free Husbandry Conditions Alleviate Behavioral Deficits and Neurodegeneration in AD10 Anti-NGF Mice
}

\author{
Stamatina Tzanoulinou ${ }^{\mathrm{a}}$, Rossella Brandi ${ }^{\mathrm{b}}$, Ivan Arisi ${ }^{\mathrm{b}}$, Mara D' Onofrio ${ }^{\mathrm{b}}$, Séverine M. Urfer $^{\mathrm{c}}$, \\ Carmen Sandi ${ }^{\mathrm{a}, 1}$, Daniel Constam ${ }^{\mathrm{c}, 1}$ and Simona Capsoni ${ }^{\mathrm{d}, *}$ \\ ${ }^{\mathrm{a}}$ Laboratory of Behavioral Genetics, School of Life Sciences, Brain Mind Institute, Ecole Polytechnique Fédérale \\ de Lausanne, Lausanne, Switzerland \\ ${ }^{\mathrm{b}}$ Genomics Facility, European Brain Research Institute, Rome, Italy \\ ${ }^{\mathrm{c}}$ Swiss Institute for Experimental Cancer Research, School of Life Sciences, Ecole Polytechnique Fédérale de \\ Lausanne, Lausanne, Switzerland \\ ${ }^{\mathrm{d}}$ Laboratory of Biology, Scuola Normale Superiore, Pisa, Italy
}

Accepted 19 August 2013

\begin{abstract}
It has been suggested that systemic infection, occurring during aging and chronic neurodegenerative diseases, can evoke an immune response that aggravates the progression of neurodegeneration and cognitive decline. It has been shown that the AD11 neurodegeneration mouse model, expressing a recombinant anti-nerve growth factor (NGF) antibody, shows a milder phenotype when housed in murine pathogen-free (MPF) conditions with respect to AD11 mice reared in conventional (CV) housing. AD10 mice, a variant of the anti-NGF AD11 model, expressing only an immunoglobulin light chain for the transgenic anti-NGF antibody, in the absence of the corresponding transgenic antibody chain $\mathrm{VH}$, exhibit a complex neurodegenerative phenotype, similar to that of AD11 mice. Here we show that the AD10 transgenic mice, housed in murine pathogen-free conditions (MPF-AD10 mice), also display a milder behavioral and neurodegenerative phenotype compared to the corresponding mice kept under conventional housing conditions (CV-AD10). As a first step toward the identification of mechanisms underlying this difference, a differential gene expression profiling was performed on brains from CV-AD10 and MPF-AD10 mice, showing a decrease of the immune response and neuroinflammation gene expression in MPF-AD10 mice. Results suggest that the activation of the immune response gene expression in the CV-AD10, in a microbially unprotected environment, might contribute to a more severe and progressive neurodegenerative phenotype, compared to the MPF mice.
\end{abstract}

Keywords: Memory deficits, murine pathogen-free, nerve growth factor, neuroinflammation, systemic inflammation

\section{INTRODUCTION}

Molecular cross-talk between the immune and nervous systems may contribute to the pathogenesis and progression of both neurodevelopmental and neurodegenerative disorders. Several studies suggest that

\footnotetext{
${ }^{1}$ These authors contributed equally to this manuscript.

*Correspondence to: Simona Capsoni, PhD, Laboratory of Biology, Scuola Normale Superiore Piazza dei Cavalieri 7, 56126 Pisa, Italy. Tel.: +39 0503153198; Fax: 0039 0503153220; E-mail: simona.capsoni@sns.it.
}

systemic infection, occurring during aging and chronic neurodegenerative diseases, can evoke an exaggerated immune response that contributes to the progression of neurodegeneration and cognitive decline [1]. The anti-NGF AD11 mouse model develops a progressive neurodegeneration as consequence of selective neutralization of mature nerve growth factor (NGF) with respect to its unprocessed precursor [2-4]. It has been shown that raising AD11 anti-NGF transgenic mice in a murine pathogen-free (MPF) condition slows the onset of neurodegeneration that they develop when raised 
in conventional housing (CV-AD11) [5]. Moreover, in the AD11 neurodegeneration model, downstream effectors of activated immune response and inflammatory pathways were also found to be regulated as one of the earliest events in the progression of neurodegeneration in CV-AD11 mice [6]. On the whole, these data revealed a complex picture of an immunotrophic and inflammatory imbalance in brain regions of AD11 mice that might have a significant role in the neurodegenerative process.

A neurodegenerative phenotype akin to that of AD11 mice develops in AD10 mice which only express the VK light chain of anti-NGF antibody [7]. In the absence of a corresponding heavy chain (VH) transgene, the VK light chain forms a hybrid antibody with endogenous mouse IgG heavy chain in lymphocytes. The production of AD10 mice takes advantage from the generation of hybrid antibodies, made from the assembly of rearranged transgenic light chains, expressed in lymphocytes, with endogenous heavy chains, from the repertoire of the host mouse. Hybrid antibodies have been well described in the context of immunological studies on Ig locus rearrangements, transcription, allelic exclusion, and production of transgenic mice [8].

In our context, AD10 mice express a functional hybrid anti-NGF antibody derived by the assembly of the exogenous anti-NGF transgenic antibody light chain (VK), with any of a number of endogenous heavy chains expressed in the host mouse lymphocytes. Since anti-NGF hybrid antibodies are formed in the absence of the corresponding transgenic antiNGF antibody heavy chain (VH), they are obligatorily formed in the lymphocytes of the mouse and therefore are primarily expressed systemically [7]. Quite unexpectedly, it was found that AD10 mice exhibit a complex neurodegenerative phenotype, similar to that of anti-NGF AD11 mice [7]. AD10 mice develop a central neurodegeneration characterized by cholinergic deficit, tau hyperphosphorylation, amyloid- $\beta$ (A $\beta)$ accumulation derived from the altered processing of endogenous mouse A $\beta P P$, and by deficits in nonspatial and spatial memory [7]. This demonstrated that the central neurodegeneration observed in AD10 mice has a peripheral, systemic origin [7], deriving from the circulating hybrid mouse-human anti-NGF antibodies [7]. In this study, we investigated whether AD10 mice, similarly to AD11 mice, when housed in pathogenfree conditions (MPF-AD10) would also show a milder neurodegeneration phenotype. We were guided by the hypothesis that since an endogenous immune system is necessary to form hybrid anti-NGF antibodies, in the form of a repertoire of heavy chains, some of which should be permissive to reconstitute a functional antiNGF antibody, when assembled with the transgenic anti-NGF light chain, the likelihood to form hybrid anti-NGF antibodies would be lower under MPF conditions, in comparison to $\mathrm{CV}$ conditions.

\section{MATERIALS AND METHODS}

\section{Anti-NGF AD10 mouse model}

AD10 mice [2] were obtained by pronuclear injection of the plasmid pcDNA-neo/VK $\alpha \mathrm{D} 11 \mathrm{HuCK}$ into zygotes of C57BL/6xSJLF2 hybrid mice [9]. This plasmid harbors the variable regions from the rat $\alpha \mathrm{D} 11$ anti-NGF antibody linked to a human $\mathrm{Ck}$ constant region (to facilitate the detection of transgenic antibodies against the background of mouse Igs), under the transcriptional control of the human cytomegalovirus (CMV) early region promoter. Analysis of transgenic mice was performed by PCR on genomic DNA from tail biopsies, as described [7]. For the analysis of a separate cohort in MPF housing conditions, AD10 mice were re-derived at Taconic and crossed to C57BL/6 females at EPFL. Heterozygous male offspring and wild-type (WT) littermates were analyzed in generations F2-F4.

\section{Housing conditions}

Mice were kept in plastic cages under a 12 hours dark to light cycle (lights on at $7.30 \mathrm{am}$ ), constant room temperature $\left(22 \pm 2{ }^{\circ} \mathrm{C}\right)$ with food and water ad libitum at the conventional facility in the European Brain Research Institute (EBRI, Rome, Italy).

For MPF conditions, we used the animal facility at EPFL, Lausanne, using the same housing conditions as for the CV-housed mice.

Experiments were performed according to the national and international laws for laboratory animal welfare and experimentation (EEC council directive 86/609, OJ L 358, 12 December 1987). All procedures performed at EPFL also complied with the Swiss National Institutional Guidelines on Animal Experimentation and approved by the Swiss Cantonal Veterinary Office Committee for Animal Experimentation.

\section{Morris water maze}

The water maze apparatus consisted of a circular pool $(140 \mathrm{~cm}$ diameter $)$ that was filled with opaque 
water. The temperature was maintained at $26^{\circ} \mathrm{C} \pm 1^{\circ} \mathrm{C}$ during the experiment. A $10 \times 10 \mathrm{~cm}^{2}$ platform was placed $1.3 \mathrm{~cm}$ below the water surface. The water maze was surrounded by clearly discernible visual cues to facilitate spatial orientation during the training phase. The experiment was divided in three phases. The habituation phase consisted of $2 \times 1$-minute trials during which mice were habituated to the room and apparatus (with the platform in the center of the pool). The pool was surrounded by curtains so that there were no visual cues available for orientation. After one day, the training phase started. Spatial learning sessions were conducted for three consecutive days and each animal was subjected to $4 \times 60$-second trials/day with an interval of 15-20 minutes between two consecutive trials. The platform remained constantly at a target quadrant. The starting point for each trial was randomly chosen. In order to assess the spatial memory of the animals, a probe trial was performed 24 hours after the last training session (day 4). During this phase, the platform was removed and the mice were allowed to swim freely for 60 seconds. The data were analyzed in blocks of two trials (B1 to B6), and the distance that the animals swam to find the platform was used as an indication of learning. The percentage of the time spent in the quadrant that contained the platform during training (target quadrant) versus the opposite quadrant was used as an index of spatial memory.

\section{Data collection and statistical analysis}

In the water maze, mice were tracked automatically with EthoVision 3.0/3.1 (Noldus, Wageningen, the Netherlands). The results were analyzed using the SPSS 13.0 statistical package and the graphs were made using GraphPad Prism 5.

The data were analyzed with analysis of variance (ANOVA) with repeated measures, Student's $t$-tests, and one-sample $t$-tests as considered appropriate. All results represent the mean \pm the standard error of the mean (SEM) and the significance was set at $p \leq 0.05$.

\section{Anti-NGF antibody detection in blood serum and brain}

The serum and brains of AD10 mice were tested for the presence of hybrid anti-NGF antibodies composed from the assembly of transgenic rat/human $\alpha \mathrm{D} 11$ light chain to mouse heavy chains. Hybrid anti-NGF antibodies were quantified using biotinylated anti-human $\mathrm{K}$ chain antibodies. Blood sera from 3 month-old WT $(n=8), \mathrm{CV}-\mathrm{AD} 10$ mice $(n=8)$, and MPF-AD10 mice $(n=13)$ were diluted $1: 10$ in $2 \%$ milk/PBS and used for ELISA in plates coated with $5 \mu \mathrm{g} / \mathrm{ml}$ of mouse NGF. The amounts of recombinant antibody found in the serum and in the brain were determined by comparison to a calibration curve. Purified $\alpha$ D11 antibody dilutions were prepared, in the range between 0.125 and $250 \mathrm{ng}$ of purified $\alpha \mathrm{D} 11$, in $2 \%$ milk/PBS containing $1: 10$ dilutions of blood serum or brain extracts from control mice, as appropriate.

\section{Immunohistochemistry and histological analysis}

Mice were anesthetized with pentobarbital to $150 \mathrm{mg} / \mathrm{kg}$ and perfused with $50 \mathrm{ml} \mathrm{4 \%}$ PFA in PBS. Brains were removed and post-fixed overnight in the same fixative, cryoprotected in $30 \%$ sucrose, and embedded for cryostat. Coronal sections $(30 \mu \mathrm{m})$ were washed with $25 \mathrm{mM}$ Tris-buffered saline $\mathrm{pH} 7.4$ containing $0.3 \%$ Triton $\mathrm{X}-100$, quenched with $3 \% \mathrm{H}_{2} \mathrm{O}_{2}$, blocked with $1 \% \mathrm{BSA}$ and labeled at $4^{\circ} \mathrm{C}$ as described using goat anti-ChAT antibody (EMD Millipore, Billerica, CA), mouse anti-4G8 (Covance, Princeton, NJ) $1: 250$, or goat anti-NH2 beta (N-18) $1: 100$ from Santa Cruz (Santa Cruz Biotechnology, Santa Cruz, CA) against amyloid A4 at 1:900 and 1:100 dilutions, respectively. Secondary antibodies were biotinylated rabbit anti-goat IgG $1: 400$ and $1: 100$, respectively (Vector Laboratories Inc., Burlingame, CA). Signal was amplified with Vectastain Elite ABC kit (Vector labs) followed by DAB-glucose oxidase (Sigma-Aldrich, St. Louis, MO) detection for ChAT, and Vectastain ABC-AP kit followed by alkaline phosphatase staining for amyloid. Images were acquired using a wide field Olympus AX70 microscope.

Images for the quantification of ChAT positive cells were obtained using the Olympus Virtual Slide Scanner VS120-SL using a 20x objective. The area corresponding to the medial septum (anatomical coordinates from Paxinos \& Franklin's Mouse Brain Atlas, [10]) was designed and specified manually and then cell counting was performed using a custom-made macro running in Fiji [11]. Cells were quantified dorsally to a line passing through the anterior commissure, which thus served as an anatomical boundary for the region of interest. For each animal ( $n=5$ for MPF-AD10; $n=5$ for MPF-WT, and $n=6$ for CV-AD10 mice), four sections of the medial septum were quantified ranging from 0.85 to $0.37 \mathrm{~mm}$ from Bregma and were matched anteroposteriorly. Additionally, the selected area was automatically measured for each section. Through this procedure, a standardized measurement was obtained by calculating the number of counted cells $/ \mathrm{mm}^{2}$ for 
each section, thus representing the average cell density in the medial septum for each animal. The number of $\mathrm{A} \beta$ clusters was determined as described [7].

\section{ELISA}

To quantify the amount of insoluble $A \beta_{1-42}$, brains from CV-AD10 $(n=6)$, MPF-WT $(n=4)$, and MPFAD10 $(n=4)$ mice were removed and homogenized as described before $[12,13]$. A sandwich ELISA was performed $[14,15]$. The monoclonal antibody $\mathrm{mAb}$ 4G8 was used as capturing antibody while the rabbit polyclonal antibody against $A \beta_{1-42}$ (Life Science Technologies Ltd, Paisley, UK) was used as detecting antibody. Brains from WT mice were processed in parallel with the transgenic tissue. The sensitivity of the ELISA was $10 \mathrm{pmol} / \mathrm{ml}$.

\section{RNA isolation and Real-Time qRT-PCR}

Hippocampal regions from 3 AD10 mice housed in MPF and of 3 age-matched AD10 mice in conventional housing were dissected from the right brain hemispheres at 1 or 3 months of age and stored in RNAlater (QIAGEN, Hilden, Germany). Total RNA was isolated using Trizol (Life Technologies, Grand Island, NY) and DNAse, by Qiagen columns. RNA concentrations were determined on a NanoDrop UVVIS. Only samples with an absorbance ratio in the range $1.8<$ OD260/OD280 $<2.0$ were selected. Quality of RNA samples was checked for integrity with the Agilent BioAnalyzer 2100 (Agilent RNA 6000 nano kit, Agilent Technologies, Santa Clara, CA): samples with a RIN index below 8.0 were discarded.

For qRT-PCR, each RNA sample was subjected to reverse transcriptase and Real-Time PCR. The mRNA for the VK antibody chains was also determined in each mouse by qRT-PCR with the following primers, specific for the $\alpha \mathrm{D} 11$ variable region antibody chains: VKs AGCAGAAGCCAGGGAAATCT, VKas TACCTTGCATACTGGGGTCC. The mRNAs for CD74 and Ccl6 were determined in each mouse with the following primers: CD74 s CTCCCTGGAGGAGAAGAAGC; CD74as TTACCGTTCTCGTCGCACTT; Ccl6s TCTTTATCCTTGTGGCTGTCC; Ccl6as TGGAGGGTTATAGCGACGAT.

\section{Microarray analysis}

The gene expression profiling was performed using the One-Color Microarray Agilent Platform (Agilent Technologies, Santa Clara, CA) according to the standard Agilent protocol. Agilent $8 \times 60 \mathrm{~K}$ whole mouse genome oligonucleotide microarray chips were used ( $\mathrm{p} / \mathrm{n}$ G4852A). Post-hybridization image acquisition was accomplished using the Agilent Scanner G2564B. Data extraction from the 20 bits TIFF images was accomplished by Agilent Feature Extraction version 10.1 software using the standard Agilent one-color gene expression extraction protocol (GE1_107_Sep09). Data filtering and analysis were performed using Agilent GeneSpring GX ver11.0 software and Microsoft Excel (Microsoft Corp., Redmond, WA). The "gProcessedSignal" data column of the Feature Extraction *.txt output files was used, containing the median signals corrected by spatial and multiplicative detrend. Data quality filtering was performed discarding features with the Feature Extraction flag gIsWellAboveBG $=0$ in any of the samples. Log2 filtered data were normalized to the 75th percentile. Functional annotations of differential gene lists were performed using the DAVID tool.

\section{RESULTS}

\section{Hybrid anti-NGF antibodies are undetectable in $M P F-A D 10$}

AD10 transgenic mice, which express only the transgenic light $\mathrm{K}$ chain from the anti-NGF antibody, are characterized by the assembly of the anti-NGF transgenic human light chains with endogenous mouse immunoglobulin heavy chains, yielding hybrid anti-NGF immunoglobulins secreted from mouse lymphocytes [7]. In AD10 mice, peripheral NGF neutralization elicits shrinkage of superior cervical ganglia (immunosympathectomy) and, as a consequence of this, peripheral anti-NGF antibodies cross the blood-brain barrier and reach the brain, generating an anti-NGF-dependent neurodegeneration, largely superimposable to that observed in AD11 mice (reviewed in [16]).

The presence or absence of hybrid anti-NGF antibodies, formed by endogenous heavy chain of IgG with the transgenic light chain of the $\alpha \mathrm{D} 11$ recombinant antibody was verified by ELISA on the sera of the AD10 mice housed in MPF and CV conditions, respectively (Fig. 1A). As expected, the CV mice express the recombinant hybrid anti-NGF antibodies while, surprisingly, the MPF-AD10 mice do not express the hybrid antibody (Fig. 1B), notwithstanding the fact that they express the transgenic anti-NGF light chain [7]. 

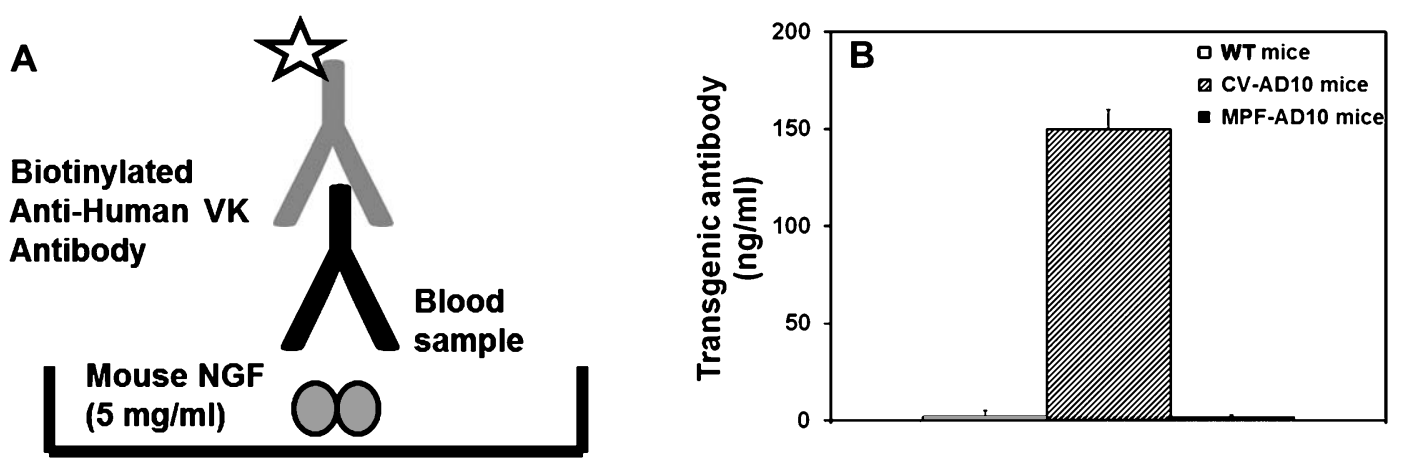

Fig. 1. The presence of hybrid antibodies obtained from the assembly of an endogenous heavy chain of IgG with the transgenic light chain of the $\alpha \mathrm{D} 11$ recombinant antibody was verified by ELISA on the sera of the AD10 mice and showed that CV-AD10 mice express hybrid anti-NGF antibodies, while MPF-AD10 mice do not express appreciable levels of hybrid anti-NGF antibodies.

This demonstrates that the activation state of the immune system has a direct consequence on the levels of hybrid anti-NGF antibodies in AD10 mice.

\section{MPF-AD10 mice are not impaired in their spatial learning and memory}

CV-AD10 mice show a progressive impairment in spatial learning and memory, as assessed by the Morris water maze test [17]. By contrast, MPF mice learned the position of the hidden platform at 6 months (Fig. 2A; $F_{4,166}=11.280, p<0.001$ ) and 9 months of age (Fig. 2B; $F_{5,75}=6.173, p<0.001$ ). Indeed, no effect of genotype was found at either 6 months $\left(F_{1,42}=0.001, p=0.980\right)$ or 9 months $\left(F_{1,15}=0.403\right.$, $p=0.535)$ of age. In the probe trial, both groups spent significantly more time swimming in the target quadrant, compared to the chance level (25\%), indicating that the spatial memory of MPF-AD10 is intact when tested at 6 months of age (Fig. $2 \mathrm{C}$; MPF-; WT: $t_{20}=2.258, p=0.035$, MPF-AD10: $t_{22}=5.624$, $p<0.001$, one-sample $t$-test) and 9 months of age (Fig. 2D; MPF-WT: $t_{7}=2.636, p=0.034$, MPF-AD10: $t_{8}=4.384, p=0.002$, one-sample $t$-test). These results support the fact that the spatial memory of the AD10 animals reared under MPF conditions remains intact, even at the age of 9 months.

\section{MPF-AD10 mice do not develop an overt neurodegeneration}

In AD11 mice reared in MPF conditions, neurodegeneration is dramatically reduced [5]. Thus, we investigated whether MPF conditions also reduce the neurodegeneration phenotype in AD10 mice. Label- ing of hippocampal coronal sections with anti-A $\beta$ antibody, at the age of 12-14 months, revealed a robust staining of amyloid plaques in 12-14 monthold CV-AD10 brains $(n=3)$, but not in age-matched MPF-AD10 $(n=5)$ (Fig. 3A-D). The absence of A $\beta$ deposition was confirmed by ELISA performed on insoluble fractions of brain extracts (Fig. 3E).

In 6-months old CV-AD10 mice, the number of choline acetyltransferase (ChAT)-immunoreactive neurons is decreased compared to the CV-WT in numbers, by a reduction of about 40-50\% ([7] and Fig. 4C). On the contrary, the number of ChAT immunoreactive neurons appeared to be normal in MPF-AD10 mice at all ages examined between 6-12 months (Fig. 4AC; $n=6 / 6$, and data not shown). Quantification of the ChAT positive cells revealed that there is no significant difference between MPF-WT and MPF-AD10 (Fig. 4C: $t_{8}=1.437, p=0.189, t$-test). These results indicate that the neurodegeneration in MPF-AD10 is less pronounced, if not absent, compared to standard housing conditions. This is in line with the fact that MPF-AD10 show negligible levels of circulating hybrid anti-NGF antibodies (Fig. 1).

\section{Global microarray differential data expression in $C V$-AD10 mice versus MPF-AD10 mice}

To gain insight into the early events induced by anti-NGF antibodies expression in AD10 mice, under MPF and CV housing conditions, we performed a gene expression microarray analysis in the hippocampus of CV- and MPF-AD10 mice at 1 and 3 months of age.

Data show that many genes are differentially expressed in the CV-AD10 compared to MPF-AD10 mice. Figure 5 presents data on the overall statistics of 

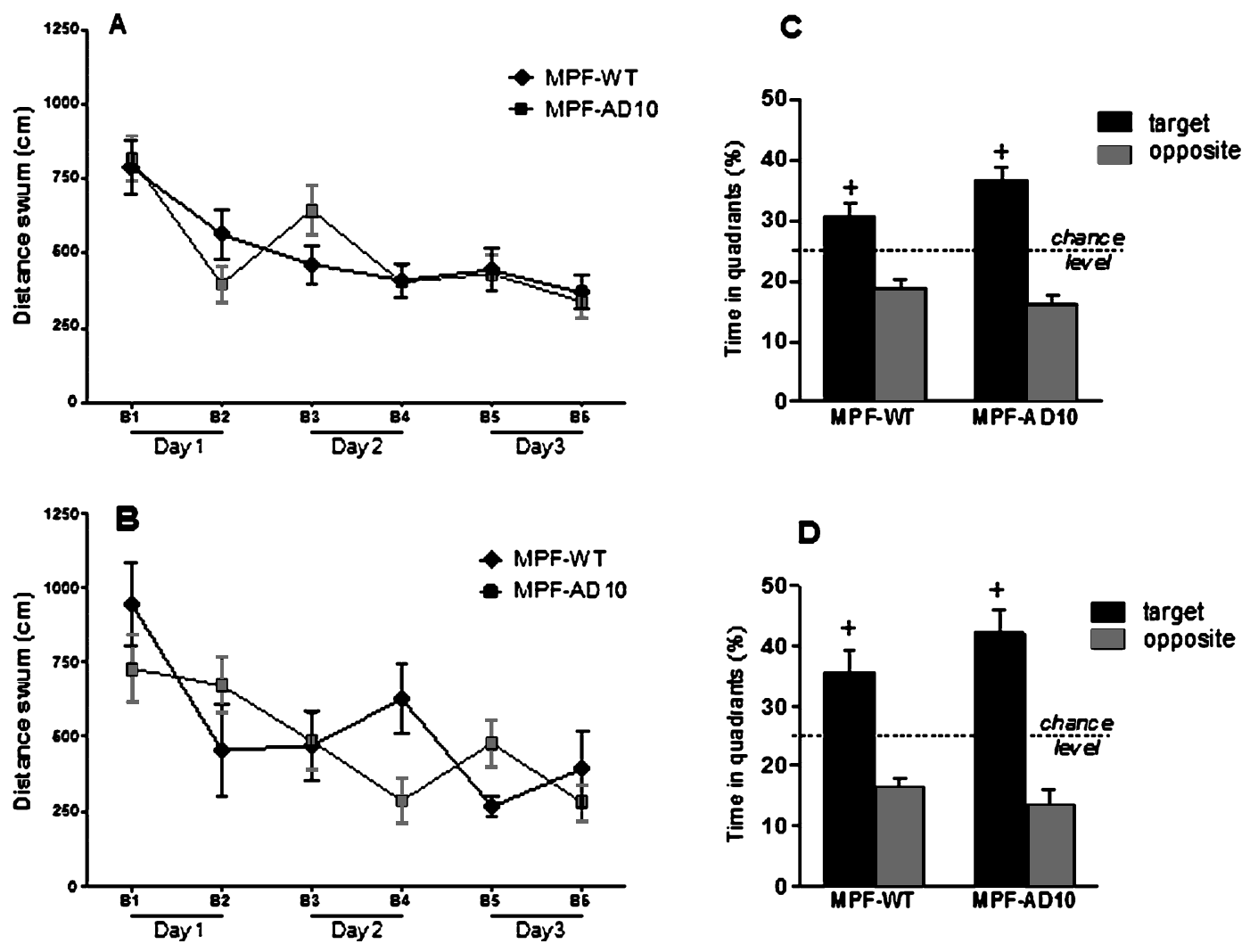

Fig. 2. Spatial learning and memory in the Morris water maze. No difference was observed between the genotypes in the acquisition phase of the water maze whether trained at 6 (A) or at 9 (B) months of age. Similarly, no differences between the two genotypes were found in the respective probe tests $(\mathrm{C}$ and $\mathrm{D})$, with both groups exhibiting good memory as indicated by their time spent in the target quadrant. Results are the mean \pm SEM $(6$ months, N: WT $=21$, MPF-AD10 $=23 ; 9$ months, N: WT $=8$, MPF-AD10 $=9)+p<0.05$ one-sample $t$-test against chance level.

the mRNAs that are differentially expressed (green for the down-regulated and red for the upregulated) in the hippocampus from CV-AD10 mice, versus the MPFAD10 mice, at 1 and 3 months of age. Data show that the total number of differentially expressed mRNAs, in CV-AD10 versus MPF-AD10 mice, is higher at 1 month, compared to the 3 months of age, suggesting a very early involvement of the hippocampus in the AD10 neurodegeneration pathogenesis of CV-AD10 mice.

\section{Differentially expressed genes in the hippocampus of $C V$-AD10 mice versus MPF-AD10 mice}

The functional analysis (Gene Ontology) of the statistically significant down-regulated and upregulated genes of the CV-AD10/MPF-AD10 at 1 and 3 months of age is reported in Figs. 6 and 7, respectively. In the expression profile of 1 month of age, the CV-AD10 group shows a striking down-regulation of gene fam- ilies related to chromatin organization and epigenetic regulation of gene expression, alongside an upregulation of gene families related to neurogenesis and neuronal development and differentiation (Fig. 6A, B).

The functional analysis of the down-regulated and upregulated genes at 3 months of age (Fig. 7A, B) shows a main regulation of the immune response genes among the selected families, with an upregulation of the major histocompatibility complex genes and other immune related genes in the CV-AD10 mice with respect to MPF-AD10 mice. The overall distribution of upregulated and down-regulated genes related to inflammatory response, as reported in Fig. 8, shows that, at 3 months, the majority (59\% of genes) of this functional category in the CV-AD10 mice is upregulated compared to the MPF-AD10 mice: there is a shift in this expression profile with age, since at 1 month the proportion of up- and down-regulated genes is very different. Comparing the number of up- and downregulated genes at the two ages in a contingency table 

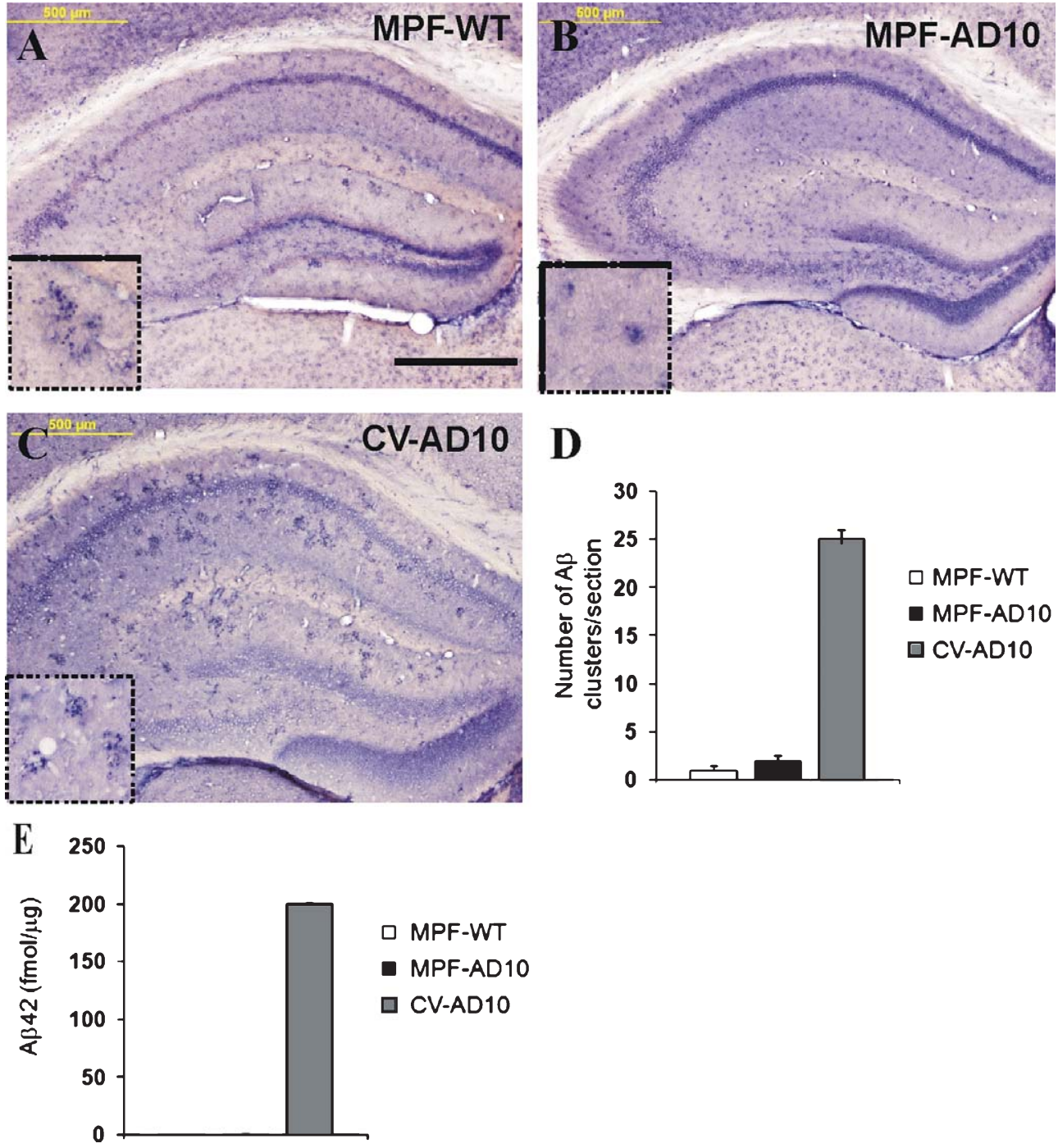

Fig. 3. Comparison of $A \beta$ neurodegeneration in AD10 mice reared in MPF or CV housing. Anti-A $\beta$ staining of (A) WT and (B) MPF-AD10 versus (C) CV-AD10 in coronal sections of the hippocampus at 12-14 months of age. (D) Quantification of A $\beta$ clusters in hippocampal sections. (E) ELISA for $A \beta_{1-42}$ formic acid extracts from WT, CV-AD10, and MPF-AD10 brains. Bars are representative of mean \pm SEM. Size bars: $500 \mu \mathrm{m}$.

by Fisher test shows a $p$-value $<10^{-3}$. The genes related to the "inflammatory response" are more upregulated $(59 \%)$ than down-regulated ones $(41 \%)$ at 3 months of age, while at 1 month $44 \%$ of this gene family are upregulated versus 56\% down-regulated (Fig. 8A, B). We defined "inflammation related" genes, the genes with the "inflammatory response" annotation in the Gene Ontology database (Supplementary Table 1).

These data show that the MPF condition changes the overall balance of the inflammatory genes and lowers the expression level of inflammation and immune related genes, thus determining a reduced neuroinflammatory phenotype. The list of the immune response
Table 1

Validation of microarray data by qRT-PCR in the hippocampus of $\mathrm{CV}$ and MPF-AD10 mice

\begin{tabular}{lcc}
\hline & qRT PCR & microarray \\
\hline CD74 & 0.89 & 0.82 \\
Cc16 & 0.89 & 0.8 \\
\hline
\end{tabular}

Values correspond to the Log2_ratio CV-AD10/MPF-AD10.

pathway genes differentially expressed at 3 months of age is shown in Supplementary Table 2, and mainly involves the upregulation of genes of the MHC complex, the classical complement activation pathway and some cytokines, while collagen alpha, heme, 

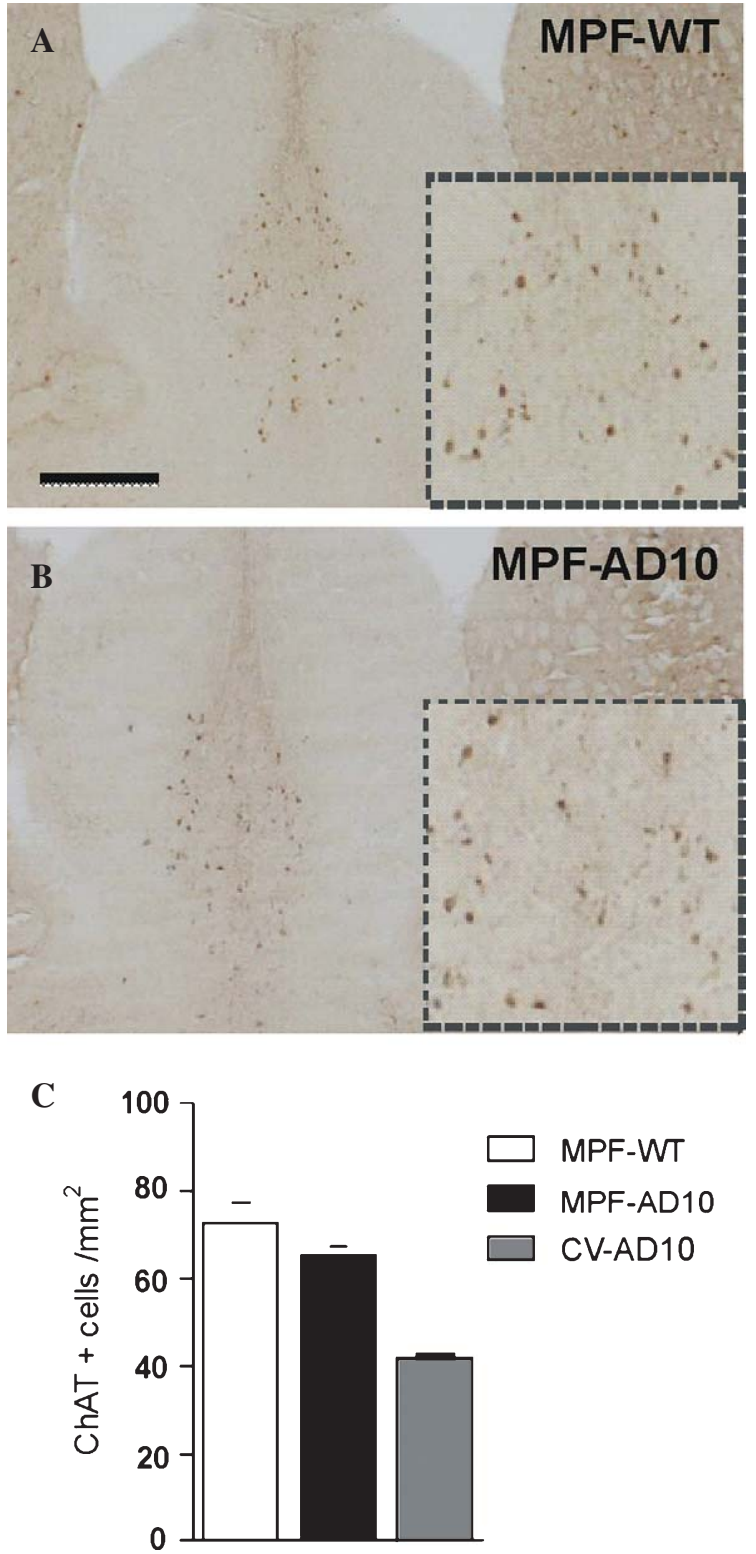

Fig. 4. Absence of cholinergic deficit in MPF-AD10 mice. AntiChAT immunostaining in the medial septum of (A) MPF-WT and (B) MPF-AD10 mice at the age of 6 months. (C) No significant difference in the number of ChAT-positive cells in the medial septum was observed between MPF-WT and MPF-AD10 at the age of 6 months, while in CV-AD10 mice a $40 \%$ decrease in the number of ChATimmunoreactive neurons can be observed. Bars are representative of mean \pm SEM. Size bars: $500 \mu \mathrm{m}$.

cytochrome families and other functional categories are down-regulated as reported in Fig. 7A, B. We verified the expression of genes that might be related to cognitive deficits induced by NGF-deprivation and found a robust down-regulation of the ChAT gene in the CV-AD10 compared to MPF-AD10 (0.4 linear scale

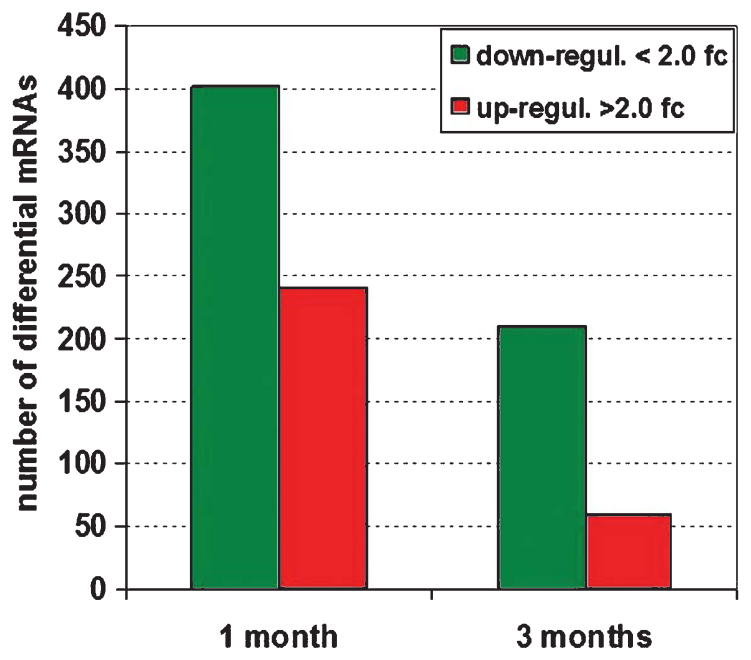

Fig. 5. Overall statistics of differentially expressed mRNAs. Upregulated (red) and down-regulated (green) mRNAs in hippocampus, at 1 and 3 months of age, of CV-AD10 versus MPF-AD10 mice. mRNAs were selected by significance analysis of microarray (SAM, $\mathrm{q}$-value $<0.05$ ) with a fc threshold of 2.0 in linear scale.

fold). This is in line with data at the immunohistochemistry level (Fig. 3 and 4) and with the fact that the decreased levels of anti-NGF antibody in the MPFAD10 determine milder cholinergic and behavioral deficits than in CV-AD10 mice.

For validation, we selected two differentially expressed genes at 3 months of age: the chemokine Ccl6 and the antigen CD74, both involved in immune system activation [18-19]. The results confirmed our previous microarray data, showing an upregulation of CD74 and of Ccl6 (Table 1). Thus, we conclude that a milder immune response is observed in MPF-AD10 mice compared to age-matched CV-AD10 mice.

\section{DISCUSSION}

In a previous article, we showed that rearing AD11 anti-NGF mice in MPF conditions slows down the progression of the neurodegeneration [5]. In this study we used another related transgenic anti-NGF mouse model, the AD10 mouse, to investigate the effects of MPF conditions on neurodegeneration and on the production of peripheral hybrid anti-NGF antibodies. We show that the expression of the hybrid anti-NGF antibody is absent in MPF-AD10 mice, despite the continuous expression of the VK light chain transgene. This is likely due to a reduced activation of the immune system under MPF conditions, leading 

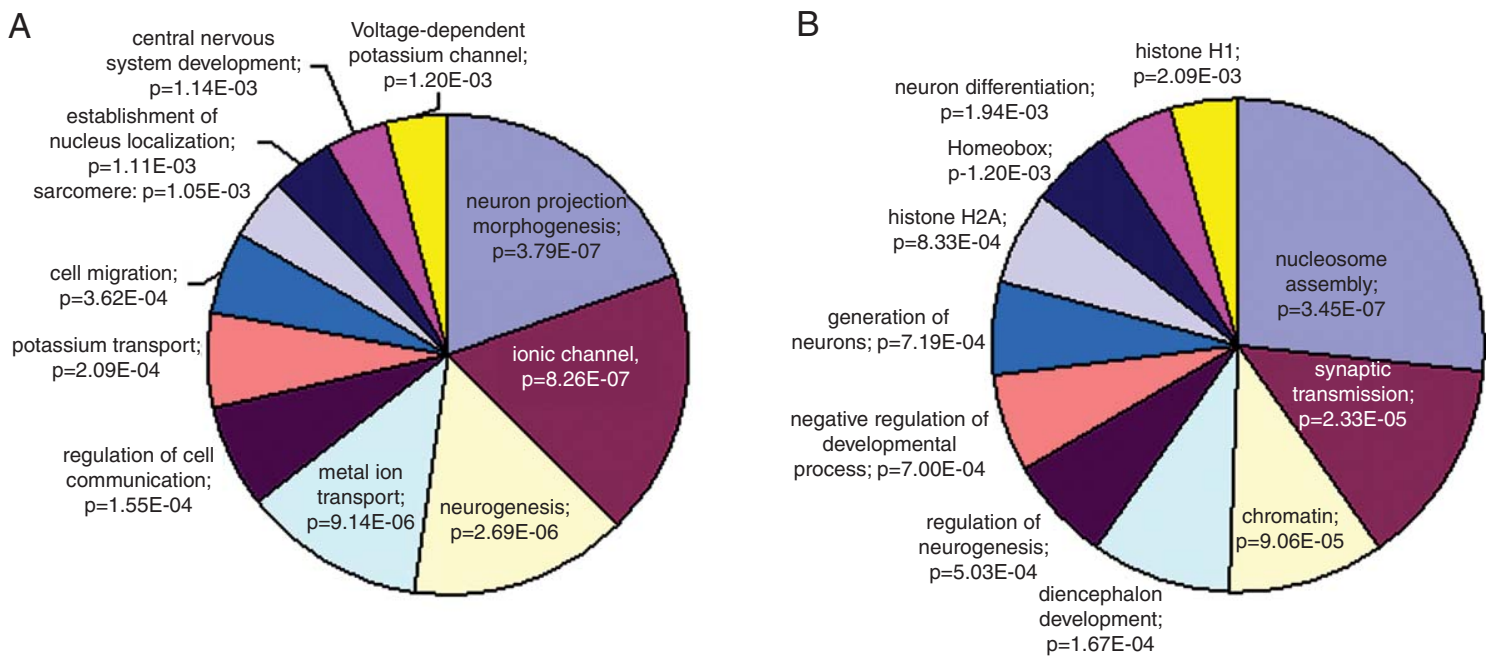

Fig. 6. Functional analysis of up- or down-regulated genes in 1-month old CV-AD10 versus MPF-AD10 mice. The genes subjected to gene ontology analysis using the cluster tool of DAVID database were upregulated $>1.5$-fold (A) or down-regulated $<1 / 1.5$ (B) in linear scale. For each cluster a representative functional term and the corresponding $p$-value are shown; $p$-value threshold is set to 0.05 . The area of each slice is proportional to $\left[-\log _{2}(\mathrm{p})\right]^{1.5}$.
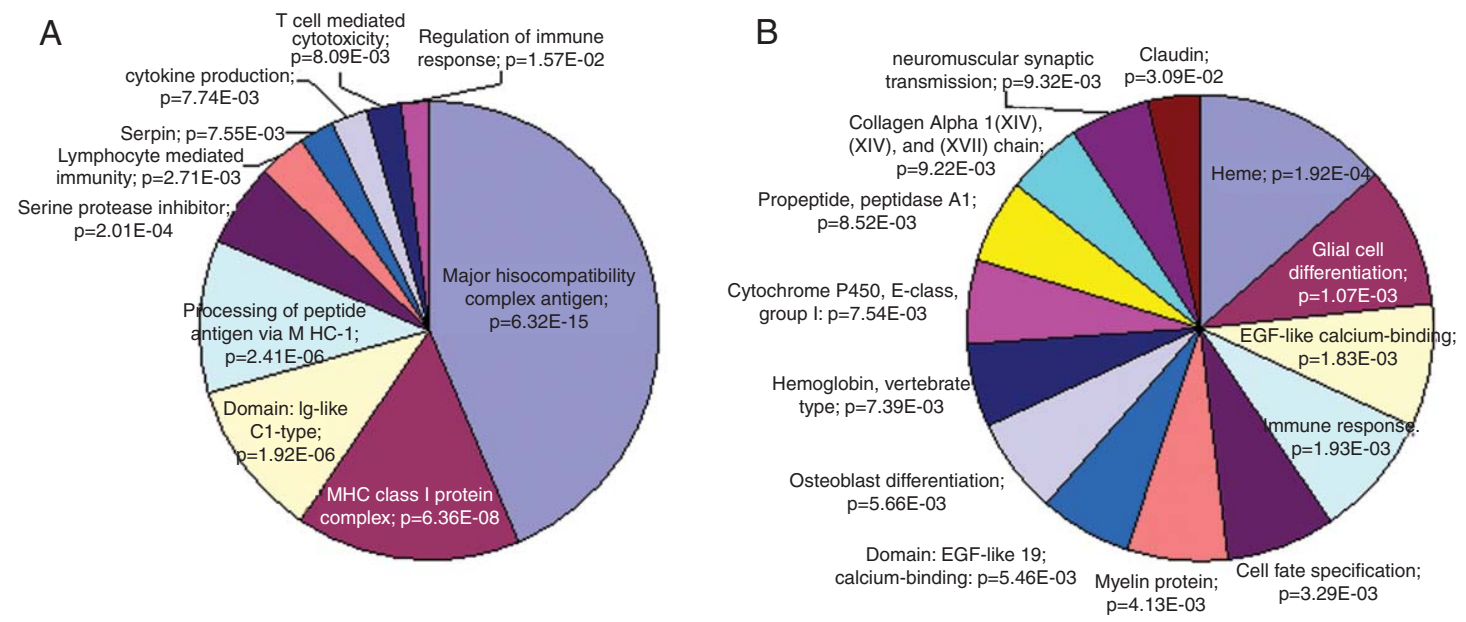

Fig. 7. Functional analysis of up- or down-regulated genes in 3-month old CV-AD10 versus MPF-AD10 mice. The genes subjected to gene ontology analysis using the cluster tool of DAVID database were upregulated $>1.5$-fold (A) or down-regulated $<1 / 1.5$ (B) in linear scale. For each cluster a representative functional term and the corresponding $p$-value are shown; $p$-value threshold is set to 0.05 . The area of each slice is proportional to $\left[-\log _{2}(\mathrm{p})\right]^{1.5}$.

to reduced diversity of the heavy chain repertoire and hence a lower capability of assembling functional antiNGF antibodies from the anti-NGF VK chain with endogenous VH chains. In AD10 the hybrid anti-NGF antibodies are a "hybrid" polyclonal mixture [7], made by one single light chain (the anti-NGF derived VK transgenic chain), combined in mouse lymphocytes with a mouse heavy chain, out of the whole repertoire of mouse heavy chains expressed by the mouse lymphocytes. Thus, in the MPF situation, the repertoire of mouse heavy chains out of which heavy chains permissive for reconstituting an anti-NGF specificity are selected, is more limited, and therefore the overall anti-NGF population is lower, due to the lower level of immune system activation. Since AD10 mice housed in MPF conditions express reduced amounts of hybrid anti-NGF antibodies, they are "protected", compared to the mice housed in the conventional one (Fig. 9). This prospects the AD10 as a model in which the overall circulating levels of functional anti-NGF antibodies is directly linked to, and regulated by, the activation levels of the immune system (Fig. 9).

There is experimental evidence that influenza and other nasal viruses can trigger an extensive but 

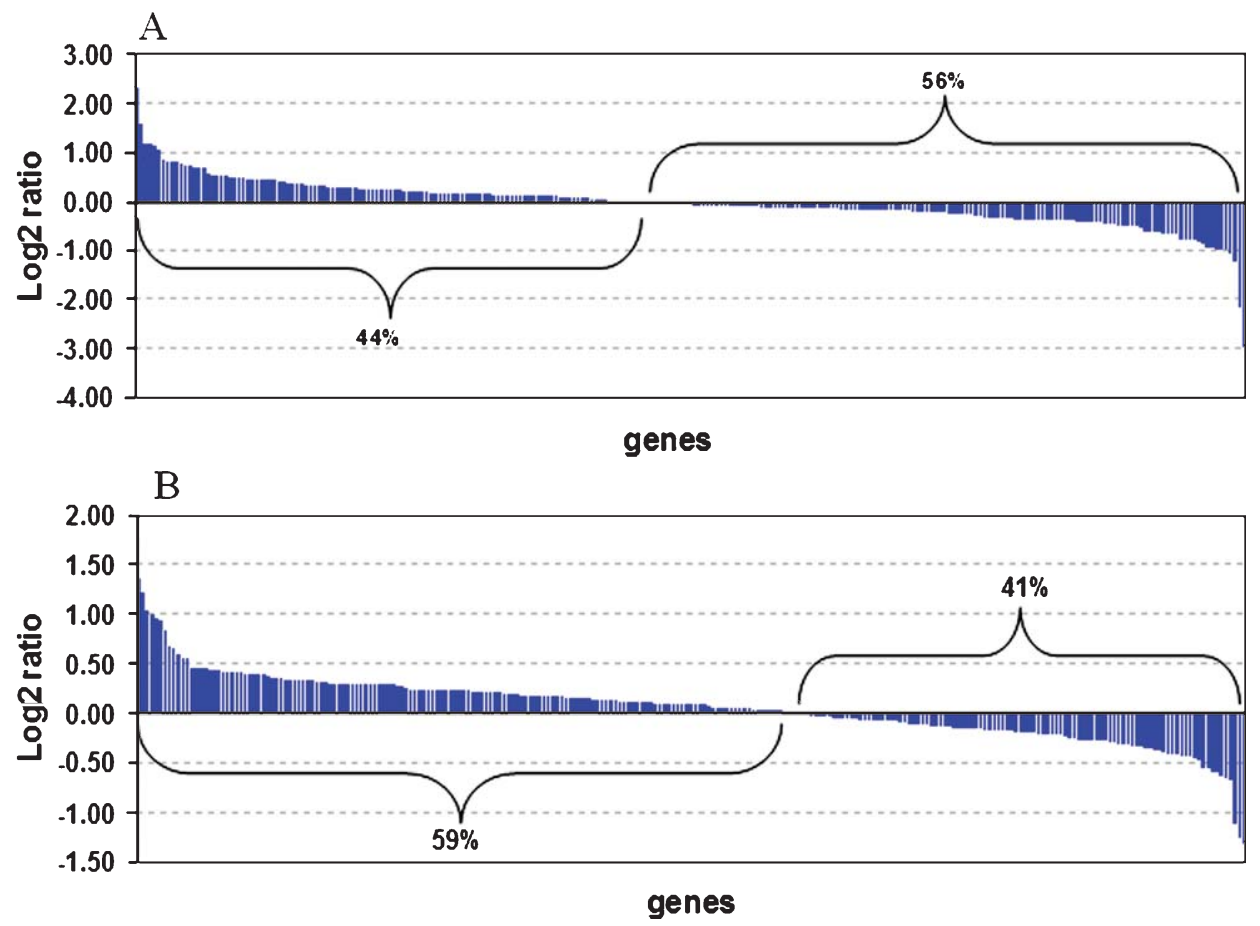

Fig. 8. Overall distribution of $\log 2$ fold change ratios of genes related to inflammatory response in CV-AD10 versus MPF-AD10 mice. Every thin blue column along the $\mathrm{x}$-axis corresponds to a gene. The gene list shown here was obtained from the AmiGO database by selecting all the gene symbols with the "inflammatory response" term (GO:0006954) annotation, at 1 month (A) and 3 months of age (B).

covert neuroinflammation [20]. The viruses invade the olfactory bulb through the olfactory nerves and then associate with glial cells that release cytokines. Epidemiological studies have associated human neurodegeneration to viral infections by common pathogens such as herpes virus, C. pneumonia, and influenza (reviewed in [20]). In a large cohort study, hippocampal volume was correlated to the level of classical inflammation biomarkers in non-demented subjects [21]. Accordingly, damage originated by numerous minor viral infections (such as influenza) with both gliotropic and neurotropic viruses over a lifetime could contribute to neurodegeneration via cumulative neuroinflammative damage [22]. This idea is further supported by a model where a systemic immune challenge even during late-gestation predisposes WT mouse offspring to pathological brain aging, cognitive decline, and amyloid and phosphorylated tau neuropathology. Furthermore the same immune challenge in adulthood is a potent driver accelerating neurodegenerative phenotype in genetically (3xTgAD) predisposed animals [23]. In a previous study we have explored the contribution of the microbial environment to the progression of neurodegeneration in AD11 mice [5], well aware of the fact that, in these mice, an inflammatory immune response is one of the earliest event in the neurodegenerative process [6]. Here we add further information, first by showing that a pathogen-free environment also alleviates the neurodegeneration in the related AD10 model, and secondly by analyzing gene expression in the brain of CV-AD10 mice compared to MPF-AD10 mice. Even though CV-AD10 animals are infected by the pathogens Norovirus, Helicobacter spp, and Trichomonas muris that are common in conventional husbandry, they show no apparent clinical signs of infectious diseases. For example, Norovirus infection is usually asymptomatic in WT and transgenic mice, except in immunocompromised animals, where it can also be lethal [24]: this suggests that Norovirus has the potential to significantly activate the immune system. This framework may be compatible with the hypothesis of covert infections that activate the immune system, as suggested by the overexpression of immune related genes in CV-AD10 compared to MPF-AD10 (see Figs. 7-8). We found that the immune response and inflammatory genes are upregulated in CV-AD10 mice compared to MPF-AD10 mice. In particular, expres- 

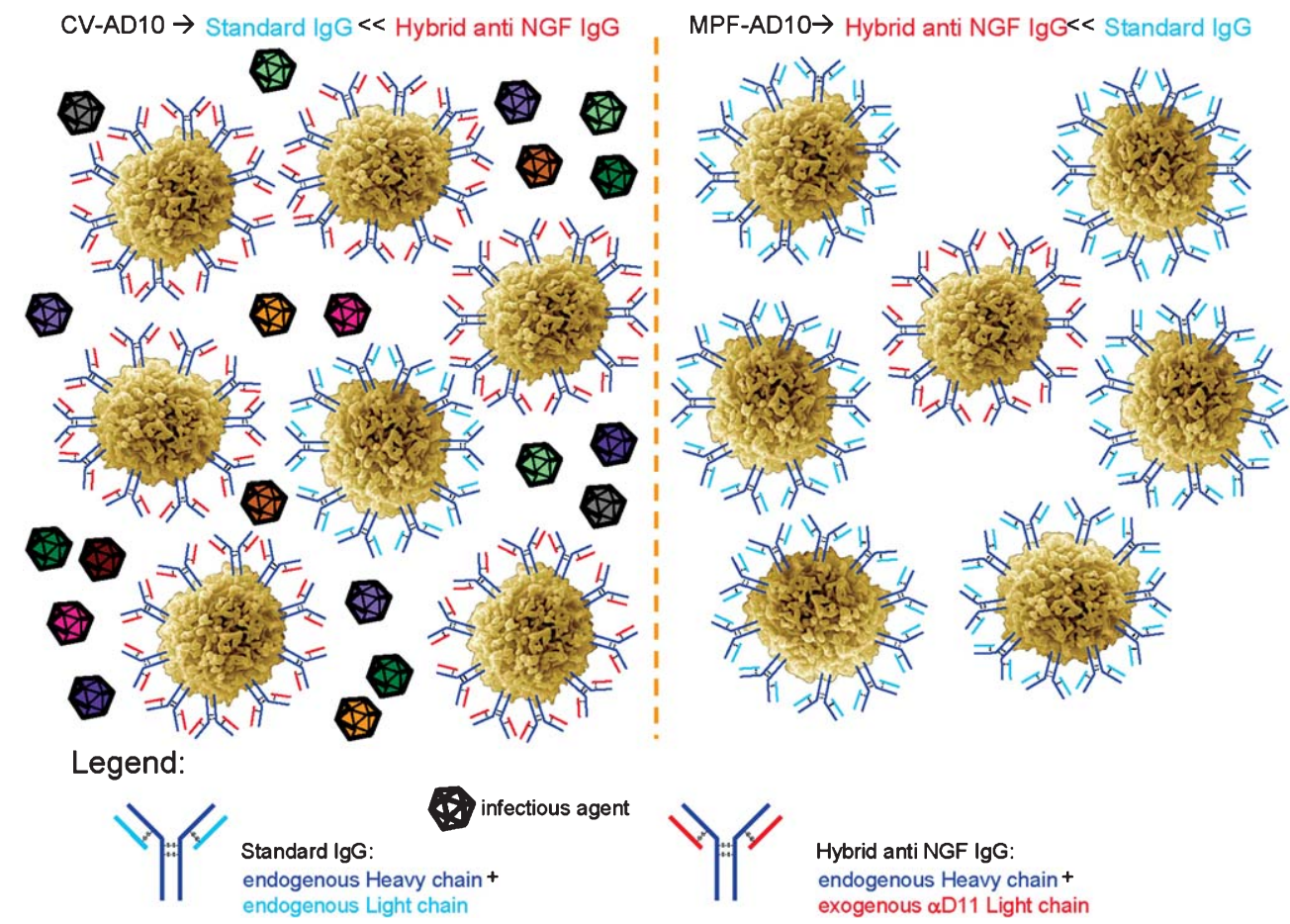

Fig. 9. Hypothesis to explain the milder neurodegenerative phenotype of MPF-AD10 mice. Lymphocytes express IgG antibodies on their surface, each cell expressing only one antigen-specific immunoglobulin type. Standard immunoglobulins, against common antigen, are depicted with the endogenous light chain colored in light blue and the endogenous heavy chain in blue. Hybrid anti-NGF immunoglobulins have the exogenous $\alpha \mathrm{D} 11$ light chain colored in red but the same type of heavy chain colored in blue. The small icosahedrons represent pathogens found in the husbandry. CV-AD10-mice live in a conventional husbandry, where a higher concentration and a larger variety of pathogen, compared to MPF husbandry, triggers a stronger immune response, globally resulting in a larger repertoire of expressed immunoglobulins. This larger repertoire in CV-AD10 mice should increase the probability of an association between the exogenous $\alpha$ D11 light chain and an endogenous heavy chain, leading to a larger number of lymphocytes expressing a hybrid anti-NGF immunoglobulin. The combination of the higher concentration hybrid anti-NGF IgGs and a greater antigen-induced infectious state could lead to the more severe neurodegenerative phenotype.

sion of the chemokine Ccl6 was increased. Ccl6 is upregulated in a variety of inflammation models such as the IL13-induced inflammation and experimental demyelinating disorders in the CNS [25]. In line with an upregulation of the immune response in CV housing conditions, we found increased expression of $\mathrm{CD} 74$, an integral membrane protein that acts both as a chaperone for MHC class II molecules as well as a receptor for macrophage migration inhibitory factor [19]. The expression of CD74 has recently been found to be increased in microglia cells in AD cases compared to age-matched controls [26].

The activation of an immune response in CVAD10 mice would increase the immune repertoire of expressed antibody genes, thereby increasing the likelihood of finding a VH heavy chain that can reconstitute a functional anti-NGF antibody with the transgenic VK chain. The fact that the regulation of the immune genes is mainly expressed only at 3 months of ages, thus only after a relatively long exposure to the same pathogens, suggests that a threshold of cumulative inflammatory response may have to be reached, in order to make the anti-NGF response a "predictive" neuropathological agent.

Besides its effects on the assembly of the hybrid antiNGF antibody, how could the inflammatory immune response influence the progression of the neurodegeneration and which is the relevance for human AD? Immune mediators, such as cytokines, can modulate synaptic transmission and alter the morphology of dendritic spines during inflammatory processes and are closely linked to plastic morphophysiological changes in neurons. It has been proposed that these immune mediators are also implicated in learning and memory processes. Indeed, our results show that the activation of the immune response genes in the CV-AD10 mice at 3 months of age, in a microbically unprotected environment, contribute to a more severe progressive 
neurodegenerative phenotype with altered cognitive performances $[7,17,27]$ compared to the MPF mice. In particular, the Major Histocompatibility Complex (MHC) class I, well known for its role in the adaptive immune response [28], regulates activity related aspects of synaptic function, during development [29, 30]. MHC I molecules are crucial for a selective maintenance of synapses during the synaptic removal process in neurons after lesion, and the lack of MHC I expression may impair the ability of neurons to regenerate axons [31]. Another immune-related molecule, the chemokine Ccl5/RANTES, has been recently proposed to be a down-regulated biomarker in a set of plasma proteins analyzed for classification and prediction of clinical AD diagnosis [32].

Besides MHC class 1 and CCl5/RANTES, the expression data at 3 months of age showed that a whole spectrum of inflammatory mediators are differentially regulated in CV-AD10/MPF-AD10, including complement component 1 and $4 \mathrm{~B}$ and other genes involved in inflammation. The relevance of the complement system in the synapse selection during the development, or the reactivation in the neurodegeneration process has previously been described [33]. The complement system is a critical element of the innate immune system recognizing and killing, or targeting for destruction, otherwise pathogenic organisms. Compelling evidence has been reported that in Alzheimer's disease complement activation occurs in the brain, and that this contributes to the development of a local inflammatory state that is correlated with cognitive dysfunction [34].

Furthermore, we found a differential regulation of some interleukin and interleukin receptor genes in the hippocampus, mainly differentially expressed at 1 month of the CV-AD10 versus the MPF-AD10. The proinflammatory cytokine interleukin- $1 \beta$ is critical for normal hippocampus-dependent cognition, whereas high levels can disrupt memory and are implicated in neurodegeneration. It has been reported that neonatal bacterial infection in rats leads to marked hippocampus-dependent memory deficits in adulthood [35]. These data implicate a long-term change within the immune system that, upon activation, impacts the neural processes underlying memory. Thus, early-life events can significantly modulate normal learningdependent cytokine activity within the hippocampus, via a specific, enduring impact on brain microglial function.

In conclusion, substantial evidence supports systemic inflammation as an epigenetic factor contributing to cognitive decline and disease progression [1]. We have shown that this is also true in neurodegeneration linked to NGF deficits ([5] and this paper). We conclude therefore that the level of systemic activation of the immune system can have a direct influence on the extent and progression of neurodegeneration. Without detracting from this conclusion, a caveat should be stated: since, in the AD10 model, the levels of the neurodegeneration-causing agent (namely hybrid antiNGF antibodies) are under the control of the activation state of the immune system, one could argue that the above-mentioned link between systemic immune system activation and the extent of neurodegeneration, in this case, has been experimentally built-in, by the way the AD10 model was constructed.

Here we suggest that the AD10 mouse model, in which the overall anti-NGF levels can be modulated by the immune system activation, might represent a good model to study a link between the immune system and neurotrophic factor activity in influencing the onset and progression of neurodegeneration.

We conclude that the level of systemic activation of the immune system can have a direct influence on the extent and progression of neurodegeneration.

\section{ACKNOWLEDGMENTS}

We would like to thank the PT-BIOp (Bioimaging and Optics Platform) of the EPFL and especially Dr. Romain Guiet for his specialized assistance and expertise for image and histological analysis. We also acknowledge Olivia Zanoletti for her excellent technical assistance and support. We thank Antonino Cattaneo (Scuola Normale Superiore Pisa, Italy) for insights and critical suggestions and for critically reading earlier versions of the manuscript. This work was funded by EU FP6 MEMORIES Project (no 037831), Fondazione Roma and FIRB (RBAP10L8TY) and by intramural funding from the EPFL.

Authors' disclosures available online (http://www.jalz.com/disclosures/view.php?id=1919).

\section{SUPPLEMENTARY MATERIAL}

Supplementary tables are available in the electronic version of this article: http://dx.doi.org/10.3233/JAD131037.

\section{REFERENCES}

[1] Perry VH (2010) Contribution of systemic inflammation to chronic neurodegeneration. Acta Neuropathol 120, 277-286. 
[2] Ruberti F, Capsoni S, Comparini A, Di Daniel E, Franzot J, Gonfloni S, Rossi G, Berardi N, Cattaneo A (2000) Phenotypic knockout of nerve growth factor in adult transgenic mice reveals severe deficits in basal forebrain cholinergic neurons, cell death in the spleen, and skeletal muscle dystrophy. J Neurosci 20, 2589-2601.

[3] Capsoni S, Ugolini G, Comparini A, Ruberti F, Berardi N, Cattaneo A (2000) Alzheimer-like neurodegeneration in aged antinerve growth factor transgenic mice. Proc Natl Acad Sci U S A 97, 6826-6831.

[4] Capsoni S, Tiveron C, Vignone D, Amato G, Cattaneo A (2010) Dissecting the involvement of tropomyosin-related kinase A and p75 neurotrophin receptor signaling in NGF deficit-induced neurodegeneration. Proc Natl Acad Sci U S A 107, 12299-12304.

[5] Capsoni S, Carucci NM, Cattaneo A (2012) Pathogen free conditions slow the onset of neurodegeneration in a mouse model of nerve growth factor deprivation. J Alzheimers Dis 31, 1-6.

[6] D’Onofrio M, Arisi I, Brandi R, Di Mambro A, Felsani A, Capsoni S, Cattaneo A (2011) Early inflammation and immune response mRNAs in the brain of AD11 anti-NGF mice. Neurobiol Aging 32, 1007-1022.

[7] Capsoni S, Tiveron C, Amato G, Vignone D, Cattaneo A (2010) Peripheral neutralization of nerve growth factor induces immunosympathectomy and central neurodegeneration in transgenic mice. J Alzheimers Dis 20, 527-546.

[8] Storb U (1987) Transgenic mice with immunoglobulin genes. Annu Rev Immunol 5, 151-174.

[9] Ruberti F, Bradbury A, Cattaneo A (1993) Cloning and expression of an anti-nerve growth factor (NGF) antibody for studies using the neuroantibody approach. Cell Mol Neurobiol 13, 559-568.

[10] Paxinos G, Franklin KBJ (2013) Paxinos and Franklin's the Mouse Brain in Stereotaxic Coordinates, 4th edition. Amsterdam: Elsevier Academic Press.

[11] Schindelin J, Arganda-Carreras I, Frise E, Kaynig V, Longair M, Pietzsch T, Preibisch S, Rueden C, Saalfeld S, Schmid B, Tinevez JY, White DJ, Hartenstein V, Eliceiri K, Tomancak P, Cardona A (2012) Fiji: An open-source platform for biological-image analysis. Nat Methods 9, 676682.

[12] Games D, Adams D, Alessandrini R, Barbour R, Berthelette P, Blackwell C, Carr T, Clemens J, Donaldson T, Gillespie F, et al. (1995) Alzheimer-type neuropathology in transgenic mice overexpressing $\mathrm{V} 717 \mathrm{~F}$ beta-amyloid precursor protein. Nature 373, 523-527.

[13] Ishihara T, Hong M, Zhang B, Nakagawa Y, Lee MK, Trojanowski JQ, Lee VM (1999) Age-dependent emergence and progression of a tauopathy in transgenic mice overexpressing the shortest human tau isoform. Neuron 24, 751-762.

[14] Capsoni S, Giannotta S, Cattaneo A (2002) Beta-amyloid plaques in a model for sporadic Alzheimer's disease based on transgenic anti-nerve growth factor antibodies. Mol Cell Neurosci 21, 15-28.

[15] Skovronsky DM, Wang J, Lee VM, Doms RW (2000) Quantifying Abeta(1-40) and Abeta (1-42) using sandwich-ELISA. Methods Mol Med 32, 79-89.

[16] Capsoni S, Brandi R, Arisi I, D’Onofrio M, Cattaneo A (2011) A dual mechanism linking NGF/proNGF imbalance and early inflammation to Alzheimer's disease neurodegeneration in the AD11 anti-NGF mouse model. CNS Neurol Disord Drug Targets 10, 635-647.

[17] Capsoni S, Amato G, Vignone D, Criscuolo C, Nykjaer A, Cattaneo A (2013) Dissecting the role of sortilin receptor signaling in neurodegeneration induced by NGF deprivation. Biochem Biophys Res Commun 431, 579-585.

[18] Coelho AL, Schaller MA, Benjamim CF, Orlofsky AZ, Hogaboam CM, Kunkel SL (2007) The chemokine CCL6 promotes innate immunity via immune cell activation and recruitment. J Immunol 179, 5474-5482.

[19] Leng L, Metz CN, Fang Y, Xu J, Donnelly S, Baugh J, Delohery T, Chen Y, Mitchell RA, Bucala R (2003) MIF signal transduction initiated by binding to CD74. J Exp Med 197, 1467-1476.

[20] De Chiara G, Marcocci ME, Sgarbanti R, Civitelli L, Ripoli C, Piacentini R, Garaci E, Grassi C, Palamara AT. Infectious agents and neurodegeneration. Mol Neurobiol 46, 614-638.

[21] Jefferson AL, Massaro JM, Wolf PA, Seshadri S, Au R, Vasan RS, Larson MG, Meigs JB, Keaney JF, Jr., Lipinska I, Kathiresan S, Benjamin EJ, DeCarli C (2007) Inflammatory biomarkers are associated with total brain volume: The Framingham Heart Study. Neurology 68, 1032-1038.

[22] Jang H, Boltz D, Sturm-Ramirez K, Shepherd KR, Jiao Y, Webster R, Smeyne RJ (2009) Highly pathogenic H5N1 influenza virus can enter the central nervous system and induce neuroinflammation and neurodegeneration. Proc Natl Acad Sci U S A 106, 14063-14068.

[23] Krstic D, Madhusudan A, Doehner J, Vogel P, Notter T, Imhof C, Manalastas A, Hilfiker M, Pfister S, Schwerdel C, Riether C, Meyer U, Knuesel I Systemic immune challenges trigger and drive Alzheimer-like neuropathology in mice. J Neuroinflammation 9, 151

[24] Ohsugi T, Matsuura K, Kawabe S, Nakamura N, Kumar JM, Wakamiya M, Morikawa S, Urano. T Natural infection of murine norovirus in conventional and specific pathogen-free laboratory mice. Front Microbiol 4, 12.

[25] Asensio VC, Lassmann S, Pagenstecher A, Steffensen SC, Henriksen SJ, Campbell IL (1999) C10 is a novel chemokine expressed in experimental inflammatory demyelinating disorders that promotes recruitment of macrophages to the central nervous system. Am J Pathol 154, 1181-1191.

[26] Bryan KJ, Zhu X, Harris PL, Perry G, Castellani RJ, Smith MA, Casadesus G (2008) Expression of CD74 is increased in neurofibrillary tangles in Alzheimer's disease. Mol Neurodegener 3, 13.

[27] Capsoni S, Carlo AS, Vignone D, Amato G, Criscuolo C, Willnow TE, Cattaneo A (2013) SorLA deficiency dissects amyloid pathology from tau and cholinergic neurodegeneration in a mouse model of Alzheimer's disease. J Alzheimers Dis 33, 357-371.

[28] Saunders PM, van Endert P (2011) Running the gauntlet: From peptide generation to antigen presentation by MHC class I. Tissue Antigens 78, 161-170.

[29] Boulanger LM, Shatz CJ (2004) Immune signalling in neural development, synaptic plasticity and disease. Nat Rev Neurosci $\mathbf{5}, 521-531$.

[30] Goddard CA, Butts DA, Shatz CJ (2007) Regulation of CNS synapses by neuronal MHC class I. Proc Natl Acad Sci U S A 104, 6828-6833.

[31] Oliveira AL, Thams S, Lidman O, Piehl F, Hokfelt T, Karre K, Linda H, Cullheim S (2004) A role for MHC class I molecules in synaptic plasticity and regeneration of neurons after axotomy. Proc Natl Acad Sci U S A 101, 17843-17848.

[32] Ray S, Britschgi M, Herbert C, Takeda-Uchimura Y, Boxer A, Blennow K, Friedman LF, Galasko DR, Jutel M, Karydas A, Kaye JA, Leszek J, Miller BL, Minthon L, Quinn JF, Rabinovici GD, Robinson WH, Sabbagh MN, So YT, Sparks DL, Tabaton M, Tinklenberg J, Yesavage JA, Tibshirani R, Wyss-Coray T (2007) Classification and prediction of clini- 
cal Alzheimer's diagnosis based on plasma signaling proteins. Nat Med 13, 1359-1362.

[33] Veerhuis R, Nielsen HM, Tenner AJ (2011) Complement in the brain. Mol Immunol 48, 1592-1603.

[34] Zabel M, Schrag M, Mueller C, Zhou W, Crofton A, Petersen F, Dickson A, Kirsch WM (2012) Assessing candidate serum biomarkers for Alzheimer's disease: A longitudinal study. J Alzheimers Dis 30, 311-321.
[35] Chong YP, Chan AS, Chan KC, Williamson NA, Lerner EC, Smithgall TE, Bjorge JD, Fujita DJ, Purcell AW, Scholz G, Mulhern TD, Cheng HC (2006) C-terminal Src kinasehomologous kinase (CHK), a unique inhibitor inactivating multiple active conformations of Src family tyrosine kinases. J Biol Chem 281, 32988-32999. 